\title{
Targeting colorectal cancer with anti-epidermal growth factor receptor antibodies: focus on panitumumab
}

\author{
Daanish Hoda' \\ George R Simon' \\ Christopher R Garrett ${ }^{1,2}$ \\ 'Division of Interdisciplinary \\ Oncology, H. Lee Moffitt Cancer \\ Center and Research Institute, \\ Tampa, FL, USA; ${ }^{2}$ Current affiliation: \\ Department of Gastrointestinal \\ Oncology, The University of Texas MD \\ Anderson Cancer Center, Houston, \\ TX, USA
}

\begin{abstract}
Panitumumab is a fully humanized monoclonal antibody with a high degree of affinity for the extracellular domain of the epidermal growth factor receptor. Phase II clinical evaluation of this drug, when administered as a single agent, in patients with metastatic colorectal cancer refractory to chemotherapy, demonstrated a modest objective radiographic response rate with acceptable toxicity; the most frequently observed side effect is rash. A randomized phase III study in subjects with chemotherapy-refractory metastatic colorectal cancer documented a progression-free survival advantage in subjects treated with panitumumab plus best supportive care versus best supportive care alone; a difference in survival was not observed, likely due to the high cross over rate. Primary tumor $K R A S$ mutation analysis performed in this study indicated that the benefit was confined to those patients whose tumors did not contain a $K R A S$ mutation. Further studies with panitumumab will be required to develop biomarkers of response and to determine if panitumumab has a role in combination with cytotoxic chemotherapy. This article summarizes the current state-of-the-science knowledge on panitumumab therapy in the treatment of advanced colorectal cancer.
\end{abstract}

Keywords: panitumumab, colorectal cancer, monoclonal antibody, epidermal growth factor receptor

\section{Introduction}

The epidermal growth factor receptor (EGFR) is one of the HER tyrosine kinase growth factor receptor family which includes her-2/neu, EGFR3, and EGFR4; its natural ligands include transforming growth factor-alpha, epidermal growth factor, amphiregulin, and epiregulin. EGFR is a transmembrane glycoprotein with an extracellular ligand binding domain in addition to an intracellular tyrosine kinase activator. Identified first in 1962, its role in cellular function has not been completely elucidated; it is known to be an important agent in cellular proliferation, angiogenesis, and protection of the cell from potentially lethal toxic insults. Once ligand binds to the EGFR it either homodimerizes (with another EGFR monomer) or heterodimerizes (with another EGFR family monomer) (Sako et al 2000). This dimerization leads to autophosphorylation of several tyrosine kinase residues with subsequent downstream activation of intracellular signaling pathways important in cell cycle growth regulation. Murine antibodies targeting EGFR, both agonist and antagonist, were generated by Drs. Gordon Sato and John Mendelsohn in the early 1980s (Mendelsohn 2002). Preclinical studies have demonstrated that these antagonist antibodies can bind to the EGFR, with a higher affinity than the natural ligands, and by doing so interrupts proliferation of human cancer cells in vivo (Kawamoto et al 1983; Sato et al 1983).

\section{Cetuximab}

In order to better understand the development of panitumumab it is helpful to know the background and clinical development of the first monoclonal antibody directed at 
the EFGR approved by the US Food and Drug Administration (FDA) for the treatment of advanced refractory colorectal cancer. The chimeric antibody cetuximab (C225, Erbitux $\left.{ }^{\mathrm{TM}}\right)$, was derived from the first murine antibody M225 and demonstrated preclinical anti-cancer activity, both in human cancer tissue culture assays and in human cancer animal xenograft models (Olverholser et al 2000). Cetuximab was demonstrated to have significant synergy with topoisomerase II inhibitors such as irinotecan; in addition, preclinically it was demonstrated that cetuximab combined with irinotecan could inhibit tumor growth in DLD-1 and HT-29 human colorectal cancer murine xenograft models, where each agent alone did not control tumor growth (Prewett et al 2002). This ability to reverse irinotecan resistance in preclinical tumor models has also been demonstrated with oxaliplatin (Prewett et al 2007). The EGFR has been demonstrated to be an important pathway in the development of colorectal cancer progression and metastasis therefore making it an attractive target for anticancer therapy in colorectal cancer (Lockhart et al 2005).

Clinical trials have subsequently shown that in subjects with metastatic colorectal cancer, refractory to at least one line of prior cytotoxic therapy, the use of cetuximab monotherapy was associated with a radiographic partial response (PR) in approximately 9\% of cases (utilizing the modified World Health Organization criteria for radiographic assessment) (Saltz et al 2004). A randomized phase II study assigning subjects to either cetuximab monotherapy or cetuximab combined with irinotecan, confined to subjects with advanced colorectal cancer refractory to prior irinotecan therapy, with tumor expression of EGFR as demonstrated by immunohistochemistry (IHC), confirmed the monotherapy activity of this drug in patients with advanced colorectal cancer with a radiographic $\mathrm{PR}$ rate of $10.8 \%$ and a combination therapy response rate of $22.9 \%$ (Cunningham et al 2004). Preclinical studies showing that EGFR inhibition by monoclonal antibody therapy in vitro can overcome chemotherapy resistance accurately predicted the clinical efficacy of monoclonal EGFR inhibitors. EGFR monoclonal antibody inhibitors are the only approved therapy to date that has been clearly been shown to reverse chemotherapy resistance clinically.

Tumor EGFR inhibition can also be achieved by the use of small-molecule receptor tyrosine kinase inhibitors. Clinical trials of EGFR inhibition by receptor tyrosine kinase inhibitors has not led to the same degree of clinical efficacy in colorectal cancer as observed with the use of EGFR-directed monoclonal antibody therapies (Keiholz et al 2005; Rothenberg et al 2005). The basis for the differences in activity observed in clinical trials between these two classes of anti-EGFR therapies (monoclonal antibodies and receptor tyrosine kinase antagnonists) is not known although it suggests that the kinase independent functions for the receptor may be important in cancer progression and another possible mechanism of activity of monocloncal anti-EGFR therapy (Cocker et al 1994; Ewald et al 2003; Weihua et al 2008).

\section{Panitumumab monotherapy in colorectal cancer}

Panitumumab (ABX-EGF, Vectibix ${ }^{\mathrm{TM}}$ ) is a fully humanized, high affinity $\operatorname{IgG}_{2}$ monoclonal antibody directed against EFGR. To reduce the immunogenicity of murine monoclonal antibodies a fully humanized monoclonal antibody was rationally developed. Cetuximab consists of $34 \%$ murine protein; the chimeric recombinant monoclonal antibodies can be immunogenic accounting for the higher rates of serious hypersensitivity reactions associated with the clinical use of cetuximab compared with panitumumab. In order to reduce the allergic reactions related to the murine element, a fully humanized monoclonal antibody directed at the EGFR, panitumumab, was developed. The major differences between cetuximab and panitumumab are illustrated in Table 1. Introducing human immunoglobulin genes into mice engineered to lack functional mouse immunoglobulin genes were used to create panitumumab. The practice for the nomenclature of the naming of monoclonal antibodies is outlined in Table 2.

Panitumumab binds to the EGFR with a higher affinity than its natural ligands to prevent receptor dimerization and subsequent intracellular autophosphorylation. Preclinical studies documented its EGFR-dependent activity in xenograft models (Yang et al 2001). A phase I study conducted in subjects with advanced refractory solid tumors expressing EGFR by IHC found the drug to be well tolerated and did not identify a maximum tolerated dose (subjects were treated up to a dose of $9.0 \mathrm{mg} / \mathrm{kg}$ administered every 21 days) (Weiner et al 2008). No hypersensitity reaction was observed in the

Table I Comparison of cetuximab and panitumumab

\begin{tabular}{lll}
\hline & Cetuximab & Panitumumab \\
\hline Molecular weight & $152.1 \mathrm{kDa}$ & $147 \mathrm{kDa}$ \\
$\%$ Murine & $34 \%$ & $0 \%$ \\
Kd EGFR & $3.4 \times 10^{-10}$ & $5 \times 10^{-11}$ \\
Half-life & $\sim 112$ hours & $\sim 180$ hours \\
Grade $3 / 4$ hypersensitivity & $3 \%$ & $1 \%$ \\
\hline
\end{tabular}


Table 2 Monoclonal antibody nomenclature (Guidelines on the Use of INNs 1997)

\begin{tabular}{lllll}
\hline & \multicolumn{1}{c}{ Target } & & Source & Suffix \\
\hline$-c o(l)-$ & colonic tumor & $-u-$ & human & \\
$-m e(l)-$ & melanoma & $-0-$ & mouse & \\
$-m a(r)-$ & mammary tumor & $-a-$ & rat & \\
$-g o(t)-$ & testicular tumor & $-e-$ & hamster & $-m a b$ \\
$-g o(v)-$ & ovarian tumor & $-i-$ & primate & \\
$-\operatorname{pr}(0)-$ & prostate tumor & $-x i-$ & chimeric & \\
$-t u(m)-$ & miscellaneous tumor & $-z u-$ & humanized & \\
\hline
\end{tabular}

Example: Ce-tu-xi-mab - tumor - chimeric

Pani-tum-u-mab - tumor - human.

97 patients treated and premedication nor a loading dose was required. The pharmacokinetics demonstrated that panitumumab could be flexibly dosed from a weekly, fortnightly or on an every-3-week schedule, making it attractive to combine with other cytotoxic chemotherapy regimes. Interestingly 5 patients obtained confirmed partial responses and 19 had stable disease on this phase I trial.

This significant clinical activity seen in this phase I dose-finding study was confirmed in a larger multicenter phase II study of panitumumab administered to subjects with metastatic, EGFR expressing tumors refractory to standard chemotherapy, at a dose of $2.5 \mathrm{mg} / \mathrm{kg}$ weekly 8 out of 9 weeks (Hecht et al 2007). Out of 148 patients treated there was a $9 \%$ objective radiographic response rate and a $29 \%$ disease stabilization rate; therapy was well tolerated with side effects mainly related to skin toxiocity, only 4 subjects withdrawing from treatment due to toxicity. One patient experienced a hypersensitivity reaction but went on to receive additional cycles of therapy with appropriate premedication.

The progression-free survival (PFS) benefit associated with the use of panitumumab in chemotherapy refractory disease has now been confirmed by a large, randomized study of patients with refractory EGFR-expressing metastatic colorectal cancer (Van Cutsem et al 2007). The trial design compared the efficacy and safety of panitumamab plus best supportive care (BSC) to BSC alone in 463 patients whose tumors had demonstrated expression of EGFR by IHC (at least $1 \%$ or more EGFR tumor cell membrane staining). Subjects on the experimental treatment arm received panitumumab $6 \mathrm{mg} / \mathrm{kg}$ by vein every two weeks until disease progression. A provision in the study was made to allow cross over of those patients who progressed on BSC alone to subsequently receive panitumumab. Seventy-five percent (174) of the subjects in the BSC alone arm crossed over to the panitumumab plus BSC arm; $67 \%$ of the 463 patients had colon cancer with the remainder having rectal cancer. All patients had received 2 or more lines of prior chemotherapy with disease progression; of note patients in whom chemotherapy was discontinued due to toxicity were not eligible for study enrollment unless that had documented disease progression while on chemotherapy. The primary study endpoint was met with a statistically significant prolongation in PFS in favor of the experimental arm. There was also noted to be a statistically significant higher radiographic response rates in the panitumumab treated group $(10 \%)$ compared with the control group ( $0 \%)$. A difference in overall survival was not observed. The effect of panitumumab on survival was likely obscured by the cross-over design of the trial; $76 \%$ of the subjects assigned to the control arm (BSC) crossed over to treatment with panitumumab upon progression. The favorable effect on PFS was observed in all patient groups irrespective of age, performance status, number of metastatic sites, number of previous treatments or levels of EGFR over-expression. Based on the PFS advantage seen with the use of panitumumab compared with BSC alone in the treatment of advanced refractory colorectal cancer, panitumumab was approved by the FDA on September 27, 2006 for the monotherapy treatment of chemotherapy-refractory metastatic colorectal cancer (FDA 2006).

The clinical outcomes and toxicity observed across clinical trials of cetuximab and panitumumab are quite similar: the main difference is the lower rate of serious (grade 3 or 4 ) hypersensitivity reactions ( $2.4 \%$ versus $0.5 \%$ ). This difference is presumably to be due to the chimeric versus human nature of the antibodies. It is not known whether the difference in $\operatorname{IgG}$ subclass between the molecules leads to a different immunologic effect on the patient receiving them or whether this effect is clinically significant.

\section{Management of panitumumab rash}

Dermopathy is the most common toxicity experienced by patients on panitumumab therapy, and all patients experience some form of skin change, the most common being dry skin (xerosis) (Mitchell et al 2007). Over $80 \%$ of patients reported on clinical trials develop a sterile acneiform dermatitis as a consequence of cutaneous EGFR inhibition. Its distribution is primarily on the face and trunk but can arise in other areas particulary those exposed to the sun. For this reason avoidance of sun exposure is an important part of managing panitumumab skin reactions. Although the rash is not infectious the disruption of the skin barrier may lead to secondary staphylococcal skin infection or impetigination. Of interest the degree of skin reaction observed appears to 
be a phamacodynamic marker of anti-EGFR efficacy; this has been observed with cetuximab in a number of different disease types in which their use has been studied (Saltz et al 2003). This observation has led to an experimental strategy of dose escalation of cetuximab therapy to rash in patients with colorectal cancer; this strategy is not currently recommended as standard of care (Tejpar et al 2007).

The management of the skin toxicity is largely empiric and supportive (Fox et al 2006). Topical steroids have been used for their anti-inflammatory properties. Systemic and topical antibiotics have been used to treat the secondary infection; the use of agents such as doxycyline may also have an immunological basis by their inhibition of neutrophic recruitment to the skin. Xerosis is treated with topical hydrating lotions. The natural history of the acneiform rash is to gradually improve after 4 to 8 weeks of panitumumab therapy; after several months of therapy nail changes (parnonychia, desquamation of the distal digits) and trichomegaly occur. Due to the initial severity of the symptoms patients experience from anti-EGFR therapies, primary prophylaxis with sun barrier creams, hydrating lotions, and topical and systemic antibiotics have been studied (Mitchell et al 2008). Given the relatively low toxicity of prophylactic therapies, and the documented amelioration of symptomatic rash, their use is recommended.

\section{Panitumumab combination therapy trials in colorectal caner}

The monotherapy efficacy and the flexibility in dosing schedule initially made panitumumab an attractive agent to combine with other cytotoxic and anti-VEGF monoclonal antibody therapies with proven efficacy in metastatic colorectal cancer. Combination phase II studies of cytotoxic chemotherapy with panitumumab have shown an acceptable toxicity profile. It has been combined safely with weekly bolus intravenous irinotecan, 5 -FU modulated by folinic acid (IFL) in addition to irinotecan, bolus 5-flurouracil (5-FU) modulated by folinic acid and protracted venous infusion 5-FU, administered every 14 days (FOLFIRI) (Hecht et al 2006). In this study patients had a good performance status $(\mathrm{ECOG} \leq 1)$ and had received no prior systemic chemotherapy for metastatic colorectal cancer. Panitumumab was given at a dose of $2.5 \mathrm{mg} / \mathrm{kg}$ to 19 patients receiving IFL and 24 patients receiving FOLFIRI. This study demonstrated that panitumumab was reasonably well tolerated as combination therapy with FOLFIRI. Over half of the subjects receiving
IFL had grade 3 or 4 diarrhea (Common Toxicity Criteria, version 2) compared with $5 \%$ of patients receiving FOLFIRI. All patients had some skin-related toxicity. A small percentage of patients treated with IFL also had hypokalemia, fatigue and nausea, which was not present in those treated with FOLFIRI. The results of this phase II trial suggested a possible role for panitumumab combined with cytotoxic chemotherapy in the first-line treatment of colorectal cancer. This was studied in 2 phase III studies evaluating the first-line use of panitumumab when combined with cytotoxic therapy alone and with combination cytotoxics and anti-VEGF monoclonal antibody therapy: the PACCE study (Panitumumab Advanced Colorectal Cancer Evaluation) and the PRIME study (Panitumumab Randomized Trial In Combination with Chemotherapy for Metastatic Colorectal Cancer to Determine Efficacy). In the PACCE study subjects with previously untreated metastatic surgically unresectable colorectal cancer received either FOLFOX4 or FOLFIRI chemotherapy (at the investigator's discretion) plus bevacizumab, and were subsequently randomized to receive to receive panitumumab or no anti-EGFR therapy (Hecht et al 2007). A pre-planned interim safety analysis of the oxaliplatin and bevacizumab-treated groups, which included 407 subjects who received panitumumab and 405 subjects in the control arm showed no difference in radiographic response rates when evaluated by external review. There was a significant difference in the medial PFS of 10.5 months in the control group versus 8.8 months in the experimental (panitumumab-treated) arm $(\mathrm{p}=0.004)$. There was also increased toxicity in the panitumab treated arm with a higher rates of grade 3/4 diarrhea, dehydration, and infection observed. Based on the data obtained at this analysis the study was discontinued prematurely. Subsequent analysis of the irinotecan-treated subjects revealed similar findings (Hecht et al 2008). Based on these data the combination of panitumab with cytotoxic chemotherapy and anti-VEGF therapy is not recommended outside of investigational trials. Primary tissue was available from the majority of subjects accrued to the PACCE trial and biomarker data from the oxaliplatin-treated subjects will be forthcoming. The results from this phase III panitumumab combination trial do not exclude future studies of panitumumab in combination with cytotoxic and/or anti-VEGF therapies particularly in biomarker-directed trials enriched for subjects most likely to respond to panitumumab therapy. In the PRIME study subjects with previously untreated metastatic colorectal cancer were randomized to receive FOLFOX4 with or without panitumumab $(6.0 \mathrm{mg} / \mathrm{kg}$ every 
2 weeks). This trial has completed accrual with acceptable safety data reported; efficacy data are anticipated to be presented in the near future (Siena et al 2008).

\section{Biomarkers of anti-EGFR monoclonal antibody therapy}

As a minority of subjects with advanced metastatic colorectal cancer have a significant objective radiographic response following monoclonal antibody anti-EGFR therapy, efforts to identify biomarkers of efficacy have been undertaken. Although initial studies were designed based on the rationale that semiquantitative receptor expression measurement by means of IHC would identify subjects most likely to benefit from anti-EGFR therapy, data from these early clinical trials did not demonstrate a correlation between degree of EGFR expression by IHC and radiographic response rate. Subsequent clinical trials have not selected subjects for inclusion based on EGFR expression by IHC; data from some of these trials have shown that radiographic responses have occurred in subjects whose tumors have been shown to be EGFR negative by IHC (Chung et al 2005). Unlike non-small cell lung cancer, activating EGFR mutations are uncommonly described in colorectal cancers (Tsuchihashi et al 2005). EGFR gene copy number has been postulated to identify those subjects with colorectal cancer more likely to benefit from antiEGFR monoclonal antibody therapy (Moroni et al 2005; Sartore-Bianchi et al 2007): additional analysis of these data suggest that a low EGFR copy number may be a better negative prognostic marker of anti-EGFR therapy. KRAS mutation status is known to be an independent adverse prognostic factor in colorectal cancer (Baurault et al 2008). Studies have shown that tumors without KRAS mutations are more likely to experience a radiographic PR or stable disease in response to EGFR monoclonal antibody therapy compared with those whose tumors had demonstrated $K R A S$ mutation, suggesting that it may also be a predictive factor for efficacy of EGFR monoclonal antibody therapy (cetuximab) in metastatic colorectal cancer (Leivre et al 2006). Retrospective analysis of the primary tumors from the previously cited phase III trial of panitumumab plus BSC versus BSC (Van Cutsem et al 2007) was undertaken to evaluate for $K R A S$ mutation status and correlate this status with clinical outcome (Amado et al 2008). This analysis revealed no benefit to the use of panitumumab, in terms of PFS, overall survival, or radiographic response rate compared with the BSC arm: the benefit was confined to those subjects whose primary tumors had wild type KRAS mutation status. These data suggest that the efficacy of panitumuab is confined to tumors with wild type KRAS mutation status: based on this data set the European regulatory authorities (EMEA) have approved the use of panitumumab in patients with advanced chemotherapy refractory tumors with wild-type $K R A S$ mutation status (EMEA 2007).

Similar analysis in patients treated with cetuximab monotherapy suggest a lack of benefit in those patients with mutant KRAS tumors. Biomarker data are now available regarding the benefit of anti-EGFR therapy in combination with cytotoxic chemotherapy. In a phase III front-line study of FOLFOX-4 with and without cetuximab (the OPUS trial) there was a benefit in progression-free survival from the addition of cetuximab to chemotherapy in those patients with wild-type $K R A S$ tumors ( 7.7 versus 7.2 months, $\mathrm{p}=0.02$ ) whereas there was no benefit in those patients with mutated KRAS (5.5 versus 8.6 months, $\mathrm{p}=0.02)$ (Bokemeyer et al 2008). The biomarker data from the CRYSTAL study (a first-line phase III study comparing FOLFIRI to FOLFIRI plus cetuximab) also showed that the clinical benefit from the addition of cetuximab therapy was limited to those patients with non-mutant KRAS tumors (Van Cutsem et al 2008).

These data suggest that the use of panitumumab should be as a single agent and limited to those patients with metastatic colorectal cancer who have wild-type $K R A S$ refractory to cytotoxic chemotherapy; panitumumab therapy is not recommended in patients whose tumors have KRAS mutations. The efficacy of panitumumab in combination therapy with cytoxic or other biologic agents is still under evaluation and is considered experimental at this time.

\section{Conclusions}

Panitumumab has been shown to be effective as monotherapy in patients with chemotherapy-refractory metastatic colorectal cancer whose tumors have wild-type $K R A S$ : it does not appear to have efficacy in those with mutant $K R A S$. Data at this time do not support its use in combination therapy. It is generally well tolerated with rash as the primary side effect. As with cetuximab the degree of skin toxicity may be a surrogate marker for anti-tumor activity. Because panitumumab is a fully human monoclonal antibody, it can be administered without hypersensitivity premedication. Continued clinical studies are warranted to determine the efficacy the combination of panitumumab with chemotherapy, as well as its role with other novel targeted agents, in patients with wild-type $K R A S$ tumors both in the metastatic and adjuvant setting. 


\section{Disclosures}

The authors have no conflicts of interest to disclose.

\section{References}

Amado RG, Wolf M, Peeters M, et al. 2008. Wild-type KRAS is required for panitumumab efficacy in patients with colorectal cancer. $J$ Clin Oncol, 26:1626-34.

Barault L, Veyrie N, Jooste V, et al. 2008. Mutations in the RAS-MAPK, $\mathrm{PI}(3) \mathrm{K}$ (phosphatidylinositol-3-OH kinase) signaling network correlate with poor survival in a population-based series of colon cancers. Int $J$ Cancer, 122:2255-9.

Bokemeyer C, Bondarenko I, Hartmann JT, et al. 2008. KRAS status and efficacy of first-line treatment of patients with metastatic colorectal cancer with FOLFOX with or without cetuximab: The OPUS experience. Proc Am Soc Clin Oncol, 26 (abstract 4000).

Chung KY, Shia J, Kemeny N, et al. 2005. Cetuximab shows activity in colorectal cancer patients with tumors that do not express the epidermal growth factor receptor by immunohistochemistry. $J$ Clin Oncol, 23:1803-10.

Cocker KJ, Staros JV, Guyer CA, et al. 1994. A kinase-negative epidermal growth factorreceptor that retains the capcity to stimulate DNA synthesis. Proc Natl Acad Sci U S A, 91:6967-71.

Cunningham D, Humblett Y, Siena S, et al. 2004. Cetuximab monotherapy and cetuximab plus irinotecan in irinotecan-refractory metastatic colorectal cancer. N Eng J Med, 351:337-45.

[EMEA] European Medicines Evaluation Agency European Public Assesment Report October. 2007. [online]. URL: http://www.emea. europa.eu/humandocs/PDFs/EPAR/vectibix/H-741-en1.pdf.

Ewald JA, Wilkinson JC, Guyer CA, et al. 2003. Ligand- and kinase activity independentcell survival mediated by the epidermal growth factor receptor expressed in 32D cells. Exp Cell Res, 282:121-31.

FDA. 2006. FDA approves Vectibix (panitumumab) to treat metastatic colorectal carcinoma [online]. URL: http://www.fda.gov/cder/Offices/ OODP/whatsnew/panitumumab.htm.

Fox LP. 2006. Pathology and management of dermatologic toxicities associated with anti-EGFR therapy. Oncology (Williston Park), 20:26-34.

Guidelines on the Use of International Nonproprietary Names (INNs) for Pharmaceutical Substances. 1997. World Health Organization [online]. pp. 27-28. URL: http://whqlibdoc.who.int/hq/1997/WHO_PHARM_ S_NOM_1570.pdf.

Hecht JR, Patnaik A, Berlin J, et al. 2007. Panitumumab montherapy in patients with previously treated metastatic colorectal cancer. Cancer, 110:980-7.

Hecht J, Posey J, Tchekmedyian E, et al. 2006. Panitumumab in combination with 5-fluorouracil, leucovorin, and Irinotecan or FOLFIRI for the first-line treatment of metastatic colorectal cancer. Gastrointestinal Cancer Symposium (abstract 237).

Hecht J. Chidiac T, Mitchell E, et al. 2007. An interim analysis of efficacy and safety from a randomized controlled trial of panitumumab with chemotherapy plus bevacizumab in metastatic colorectal cancer. Ninth World Congress on Gastrointestinal Cancer (abstract 9).

Hecht JR, Mitchell E, Chidiac T, et al. 2008. Interim results from PACCE: irinotecan/bevacizumab \pm panitumumab as first-line treatment for metastatic colorectal cancer. Gastrointestinal Cancer Symposium (abstract 279).

Kawamoto TK, Sato JD, Le A, et al. 1983 Growth stimulation of A431 cells by epidermal growth factor: identification of high-affinity receptors for epidermal growth factor by an anti-receptor monoclonal antibody. Proc Natl Acad Sci U S A, 80:1337-41.

Keiholz U, Arnold D, Niederle N, et al. 2005. Erlotinib as second and third line montherapy in patients with metastatic colorectal cancer. Results of a multicenter two-cohort phase II trial. Proc Am Soc Clin Oncol, 23(abstract 3575).
Lievre A, Bachet JB, Le Corre D, et al. 2006. KRAS mutation status is predictive of response to cetuximab therapy in colorectal cancer. Cancer Res, 66:3992-5

Lockhart AC, Berlin JD. 2005. The epidermal growth factor as a receptor target for colorectal cancer therapy. Semin Oncol, 32:52-60.

Mendelsohn J. 2002. Targeting the epidermal growth factor receptor for cancer therapy. J Clin Oncol, 20:1-13s.

Mitchell EP, Perez-Soler R, Van Cutsem E, et al. 2007. Clinical presentation and pathophysiology of EGFRI dermatologic toxicities. Oncology (Williston Park), 21:4-9.

Mitchell EP, LaCouture M, Shearer H, et al. 2008. A phase II, openlabel, randomized clinical trial of skin toxicity evaluation (STEPP) in metastatic colorectal cancer patients receiving second-line FOLFIRI or irinotecan-only chemotherapy with panitumumab: early analysis. Proceedings ASCO Gastrointestinal Cancer Symposium (abstract 462).

Moroni M, Veronese S, Benvenuti S, et al. 2005. Gene copy number for epidermal growth factor receptor (EGFR) and clinical response to anti-EGFR treatment in colorectal cancer: a cohort study. Lancet Oncol, 6:257-8.

Overholser JP, Prewett MC, Hooper AT, et al. 2000 Epidermal growth factor receptor blockade by antibody IMC-C225 inhibits growth of a human pancreatic carcinoma xenograft in nude mice. Cancer, 89:74-82.

Prewett M, Hooper A, Bassi R, et al. 2002. Enhanced antitumor activity of anti-epidermal growth factor receptor monoclonal antibody IMC-C225 in combination with irinotecan (CPT-11) against human colorectal tumor xenografts. Clin Cancer Res, 8:994-1003.

Prewett M, Deevi DS, Bassi R, et al. 2007. Tumors established with cell lines selected for oxaliplatin resistance respond to oxaliplatin if combined with cetuximab. Clin Cancer Res, 13:7432-40.

Rothenberg ML, LaFleur B, Levy DE, et al. 2005. Randomized phase II trial of the clinical and biological effects of two doses levels of gefitinib in patients with recurrent colorectal cancer. J Clin Oncol, 23:9265-74.

Sako Y, Minoghchi S, Yanagida T, 2000. Single-molecule imaging of EGFR signaling on the surface of living cells. Nat Cell Biol, 2:168-172.

Saltz L, Keis M, Abbruzzese JL, et al. 2003. The presence and intensity of the cetuximab-induced acne-like rash predicts increased survival in studies across multiple malignancies. Proc Am Soc Clin Oncol, 22(abstract 817).

Saltz LB, Meropol NJ, Loehrer PJ Sr, et al. 2004. Phase II trial of cetuximab in patients with refractory colorectal cancer that express the epidermal growth factor receptor. J Clin Oncol, 22:1201-8.

Siena S, Tabernero J, Burkes RL, et al. 2008. Phase III study (PRIME/20050203) of panitumumab with FOLFOX compared with FOLFOX alone in patients with previously untreated metastatic colorectal cancer: pooled safety data. Proc Am Soc Clin Oncol, 25(abstract 4034).

Sartore-Bianchi A, Moroni M, Veronese S, et al. 2007. Epidermal growth factor receptor gene copy number and clinical outcome of metastatic colorectal cancer treated with panitumumab. J Clin Oncol, 25:3238-45.

Sato JD, Kawamoto, Le AD, et al. 1983. Biologic effects in vitro of monoclonal antibodies to human epidermal growth factor receptors. Mol Biol Med, 1:511-29.

Tejpar S, Peeters M, Humblet Y et al. 2007. Phase I/II study of cetuximab dose-escalation in patients with metastatic colorectal cancer with no or slight skin reactions on cetuximab standard dose treatment (EVEREST): pharmacokinetic, pharmacodynamic and efficacy data. Proc Am Soc Clin Oncol, 25(abstract 4037).

Tsuchihashi Z, Khambata-Ford S, Hanna N, et al. 2005. Responsiveness to cetuximab without mutations in EGFR. $N$ Engl J Med, 353:208-9.

Van Cutsem E, Peeters M, Siena S, et al. 2007. Open-label phase III trial of panitumumab plus best supportive care compared with best supportive care alone in patients with chemotherapy-refractory metastatic colorectal cancer. J Clin Oncol, 25: 1658-64. 
Van Cutsem E, Lang I, D'haens G, et al. 2008. KRAS status and efficacy in the first-line treatment of patients with metastatic colorectal cancer treated with FOLFIRI with or without cetuximab: the CRYSTAL experience. Proc Am Soc Clin Oncol, 26(abstract 2).

Weihua Z, Tsan R, Huang WC, et al. 2008. Survival of cancer cells is maintained by \EGFR independent of its kinase activity. Cancer Cell, 13:385-93.
Weiner LM, Belldegrun AS, Crawford J, et al. 2008. Dose and schedule study of panitumumab monotherapy in patients with advanced solid malignancies. Clin Cancer Res, 14:502-8.

Yang XD, Jia XC, Corvalan JR, et al. 2001. Development of ABX-EGF, a fully human anti-EGF receptor monoclonal antibody, for cancer therapy. Crit Rev Oncol Hematol, 38:17-23. 
